Received 5 September 2017

Accepted 15 September 2017

Edited by H. Stoeckli-Evans, University of Neuchâtel, Switzerland

Keywords: molecular structure; disorder; conformation; hydrogen bonding; supramolecular assembly; crystal structure.

CCDC reference: 1574718

Supporting information: this article has supporting information at journals.iucr.org/e

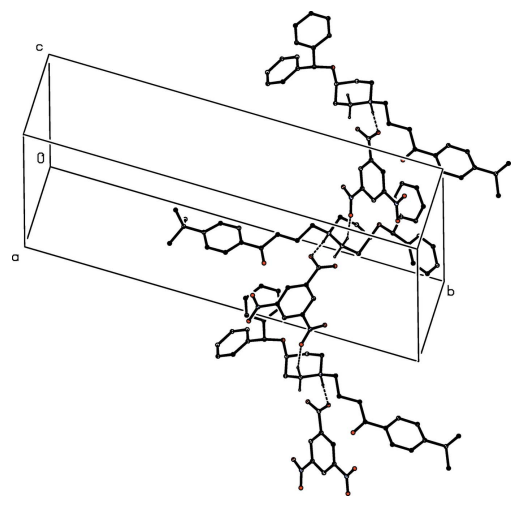

OPEN $\odot$ ACCESS

\section{Crystal structure of ebastinium 3,5-dinitrobenzoate}

Mohammed A. E. Shaibah, ${ }^{a}$ Belakavadi K. Sagar, ${ }^{a}$ Hemmige S. Yathirajan, ${ }^{a} *$ S. Madan Kumar ${ }^{\mathrm{b}}$ and Christopher Glidewell ${ }^{\mathrm{C}}$

${ }^{\mathbf{a}}$ Department of Studies in Chemistry, University of Mysore, Manasagangotri, Mysuru-570 006, India, ${ }^{\mathbf{b}}$ Department of Studies in Chemistry, Mangalore University, Mangalagangotri-574 199, India, and ' School of Chemistry, University of St Andrews, St Andrews, Fife KY16 9ST, UK. *Correspondence e-mail: yathirajan@hotmail.com

Ebastine, 4-(benzhydryloxy)-1-[4-(4-tert-butylphenyl)-4-oxobutyl]piperidine, reacts with 3,5-dinitrobenzoic acid in methanol solution to give the title 1:1 salt, ebastinium 3,5-dinitrobenzoate, $\mathrm{C}_{32} \mathrm{H}_{40} \mathrm{NO}_{2}{ }^{+} \cdot \mathrm{C}_{7} \mathrm{H}_{3} \mathrm{~N}_{2} \mathrm{O}_{6}{ }^{-}$. In the cation, the disubstituted aryl ring exhibits orientational disorder over two sets of atomic sites having occupancies 0.706 (4) and $0.294(6)$, with a dihedral angle of $41.2(5)^{\circ}$ between the two orientations: the bulky $\mathrm{Ph}_{2} \mathrm{CH}-\mathrm{O}-$ substituent occupies an axial site on the piperidine ring. The two ions in the selected asymmetric unit are linked by a nearly linear $\mathrm{N}-\mathrm{H} \cdots \mathrm{O}$ hydrogen bond and this, in combination with two $\mathrm{C}-\mathrm{H} \cdots \mathrm{O}$ hydrogen bonds, links the ions into complex sheets.

\section{Chemical context}

Ebastine, or 4-(benzhydryloxy)-1-[4-(4-tert-butylphenyl)-4oxobutyl]piperidine, is a non-sedating second generation $\mathrm{H}_{1}$ receptor antagonist, which is effective in the treatment of both allergic rhinitis, whether seasonal or perennial, and chronic idiopathic urticaria (Wiseman \& Faulds, 1996; Van Cauwenberge et al., 2004). The structure of ebastine has been the subject of two recent reports (Cheng et al., 2005: Sharma et al., 2015). Herein, we report the molecular and supramolecular structure of the 1:1 salt ebastinium 3,5-dinitrobenzoate (I), formed in the reaction between ebastine and 3,5-dinitrobenzoic acid.<smiles></smiles>

\section{Structural commentary}

The title compound (I), consists of an N-protonated ebastinium cation and a 3,5-dinitrobenzoate anion (Fig. 1), which 


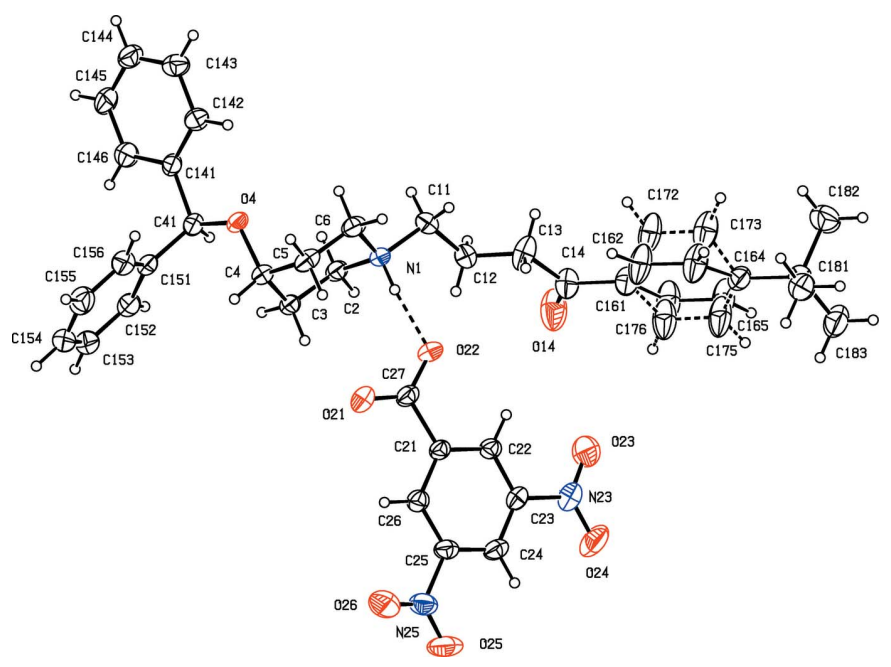

Figure 1

The molecular structure of the ionic components of compound (I), showing the atom-labelling scheme, the $\mathrm{N}-\mathrm{H} \cdots \mathrm{O}$ hydrogen bond within the selected asymmetric unit, and the orientational disorder of the disubstituted aryl ring (the major component is drawn with full lines and the minor component with broken lines). Displacement ellipsoids are drawn at the $30 \%$ probability level and, for clarity, a few of the atom labels have been omitted.

are linked within the selected asymmetric unit a by a fairly short and nearly linear $\mathrm{N}-\mathrm{H}$... O hydrogen bond (Fig. 1, Table 1). The disubstituted aryl ring in the cation is disordered over two sets of atomic sites having occupancies 0.706 (4) for the major ring orientation, labelled C161-C166, and 0.294 (4) for the minor orientation, labeled C171-C176: the dihedral angle between these two ring planes is $41.2(5)^{\circ}$ (Fig. 1). The piperidine ring adopts an almost perfect chair conformation, with a ring-puckering angle, calculated for the atom sequence $(\mathrm{N} 1, \mathrm{C} 2, \mathrm{C} 3, \mathrm{C} 4, \mathrm{C} 5, \mathrm{C} 6)$ of $\theta=0.0(3)^{\circ}$, identical within experimental uncertainty to the idealized value for a perfect chair form of $\theta=0.0^{\circ}$ (Boeyens, 1978). However, although the non$\mathrm{H}$ substituent at atom $\mathrm{N} 1$ in the ring occupies an equatorial site, as expected, the bulky $\mathrm{Ph}_{2} \mathrm{CHO}$ substituent at atom $\mathrm{C} 4$ unexpectedly occupies an axial site. This observation is the more surprising since in ebastine itself, both non-H substituents on the piperidine ring occupy equatorial sites (Cheng et al., 2005: Sharma et al., 2015). The 3,5-dinitrobenzoate anion in compound (I) is nearly planar: the dihedral angles between the aryl ring and the substituents at atoms C21, C23 and C25 are 1.4 (2), 4.2 (2) and $10.7(2)^{\circ}$, respectively: only the $\mathrm{O}$ atoms of the 5-nitro group are significantly displaced from the mean plane of the anion as a whole, 0.219 (2) $\AA$ for atom O25 and 0.187 (2) $\AA$ for atom O26: the r.m.s. deviation from the mean plane for the entire anion is only $0.082 \AA$.

\section{Supramolecular features}

In addition to the $\mathrm{N}-\mathrm{H} \cdots \mathrm{O}$ hydrogen bond within the selected asymmetric unit, already noted (cf. Fig. 1 and Table 1 ), there are two $\mathrm{C}-\mathrm{H}$.. O hydrogen bonds in the crystal of compound (I), which link the components into complex sheets,
Table 1

Hydrogen-bond geometry $\left(\AA,^{\circ}\right)$.

\begin{tabular}{lllll}
\hline$D-\mathrm{H} \cdots A$ & $D-\mathrm{H}$ & $\mathrm{H} \cdots A$ & $D \cdots A$ & $D-\mathrm{H} \cdots A$ \\
\hline $\mathrm{N} 1-\mathrm{H} 1 \cdots \mathrm{O} 22$ & $0.99(3)$ & $1.66(2)$ & $2.634(3)$ & $167(2)$ \\
$\mathrm{C} 2-\mathrm{H} 2 A \cdots \mathrm{O} 25^{\mathrm{i}}$ & 0.97 & 2.50 & $3.444(3)$ & 163 \\
$\mathrm{C} 11-\mathrm{H} 11 A \cdots \mathrm{O} 14^{\mathrm{ii}}$ & 0.97 & 2.49 & $3.358(4)$ & 150 \\
\hline
\end{tabular}

Symmetry codes: (i) $x-1,-y+\frac{3}{2}, z+\frac{1}{2}$; (ii) $x-1, y, z$.

whose formation can, however, be readily analysed in terms of two simple, one-dimensional sub-structures (Ferguson et al., 1998a,b; Gregson et al., 2000). In the simpler of the two substructures, cations related by translation are linked by a single $\mathrm{C}-\mathrm{H} \cdots \mathrm{O}$ hydrogen bond to form a $C(6)$ chain running parallel to the [100] direction (Fig. 2, Table 1). The second substructure involves the cations and the anions, and a combination of the $\mathrm{N}-\mathrm{H} \cdots \mathrm{O}$ hydrogen bond and a second $\mathrm{C}-$ $\mathrm{H}$. . O hydrogen bond links ions related by a $c$-glide plane into a $C_{2}^{2}(11)$ chain, running parallel to the [201] direction, in which cations and anions alternate (Fig. 3, Table 1). The combination of these two chain motifs generates a sheet lying parallel to (010) in the domain $0.5<y<1.0$, and a second such sheet, related to the first by inversion, lies in the domain $0.0<y<0.5$, but there are no direction-specific interactions between adjacent sheets. It is interesting to note that none of the hydrogen

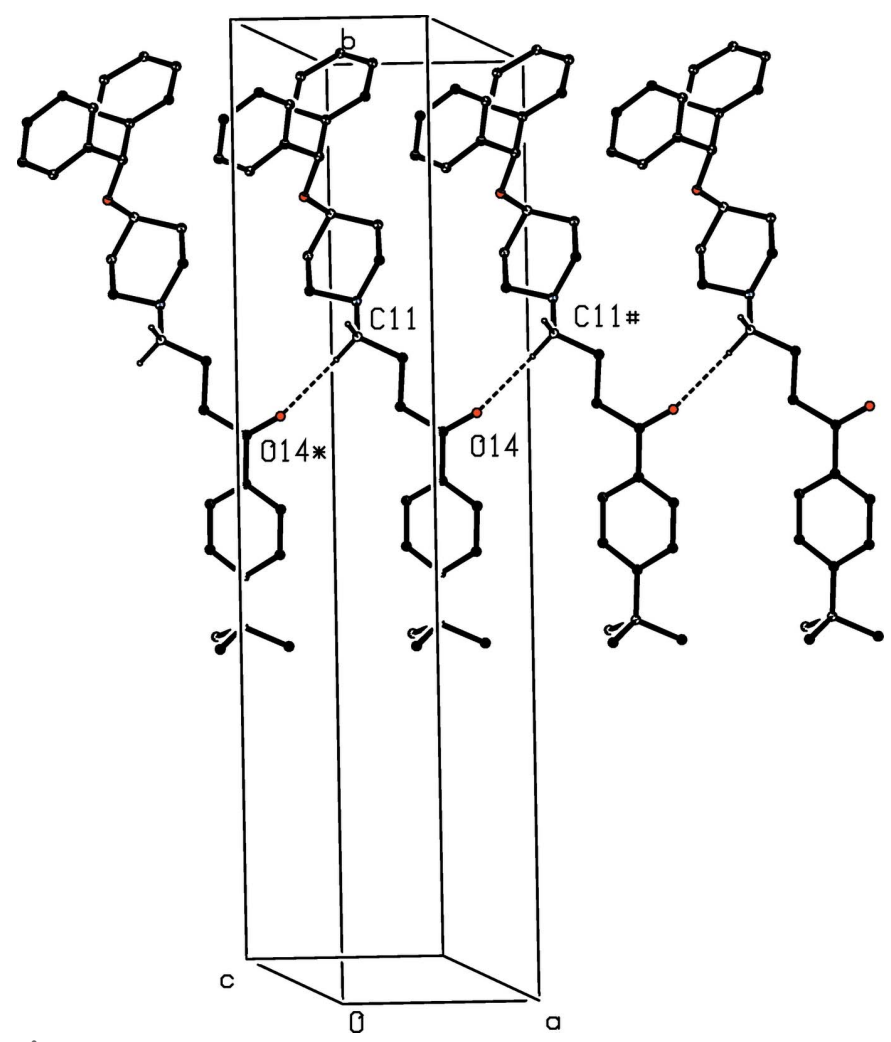

Figure 2

Part of the crystal structure of compound (I), showing a hydrogenbonded $C(6)$ chain of cations running parallel to [100]. For clarity, the anions, the minor disorder component of the cation, and the $\mathrm{H}$ atoms bonded to carrier atoms not involved in the motif shown have been omitted. The atoms marked with an asterisk $(*)$ or a hash $(\#)$ are at the symmetry positions $(-1+x, y, z)$ and $(1+x, y, z)$ respectively. 


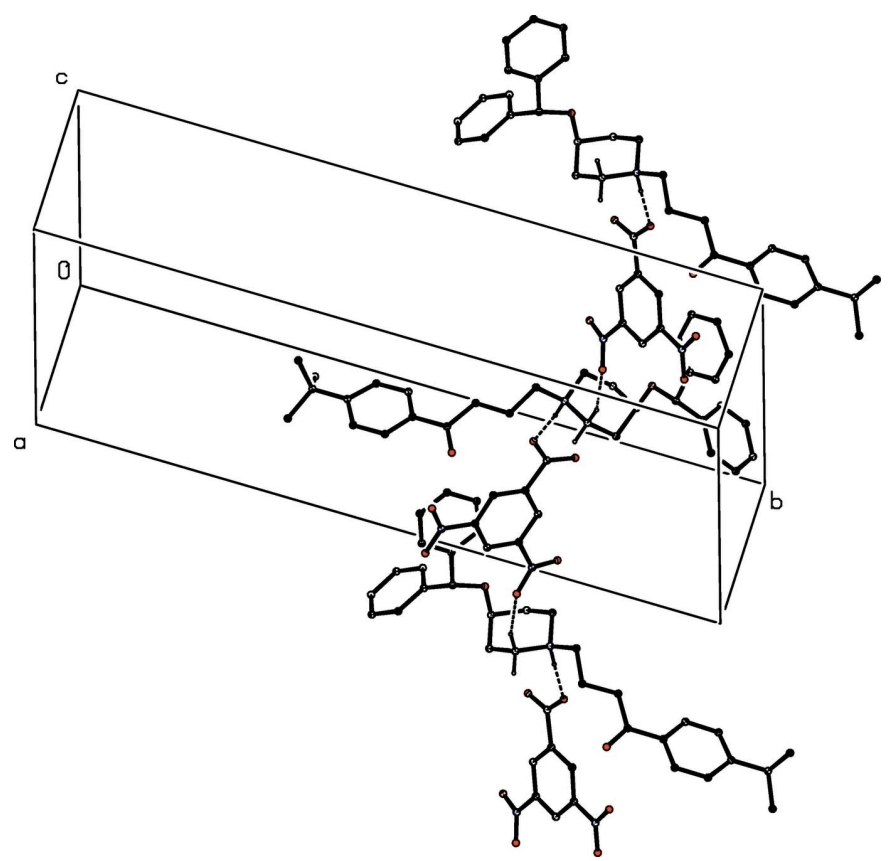

Figure 3

Part of the crystal structure of compound (I), showing a hydrogenbonded $C_{2}^{2}(11)$ chain running parallel to [201]. For clarity, the minor disorder component of the cation, and the $\mathrm{H}$ atoms bonded to $\mathrm{C}$ atoms not involved in the motif shown have been omitted.

bonds in compound (I) involves the $\mathrm{Ph}_{2} \mathrm{CHO}$ substituent, so that direction-specific interactions cannot be held responsible for the location of this substituent at an axial site on the piperidine ring.

\section{Database survey}

The molecular structure of neutral ebastine (Cheng et al., 2005; Sharma et al., 2015) differs from that of the ebastinium cation in compound (I) in two significant respects. Firstly, there is no disorder in the neutral compound as opposed to the orientation disorder of the disubstituted aryl ring in (I) and secondly, both of the non-H substituents on the piperidine ring occupy equatorial sites in the neutral compound as opposed to the presence of one axial and one equatorial substituent in (I). Neither of the two reports on the structure of ebastine gave any description of the supramolecular assembly: one (Cheng et al., 2005) noted the presence of hydrogen bonds, but the second (Sharma et al., 2015) did not record these. Accordingly, we have now examined the supramolecular assembly of ebastine using the most recently reported atomic coordinates (Sharma et al., 2015): a combination of one $\mathrm{C}-\mathrm{H} \cdots \mathrm{N}$ hydrogen bond and one $\mathrm{C}-\mathrm{H} \cdots \mathrm{O}$ hydrogen bond links the molecules into sheets lying parallel to (100) and containing $R_{2}^{2}(20)$ and $R_{6}^{6}(48)$ rings, both centrosymmetric, arranges in chess board fashion (Fig. 4). Structures have also been reported recently for some structurally related compounds with pharmacological activity, including the picrate salt of the anticholinergic drug propiverine, 4-(2,2-diphenyl-2-propoxyacetoxy)-1-methylpiperidin-1-ium picrate (Jasinski et al.,
2009), and the anti-spasmodic drug pargeverine, $N, N$ dimethyl-[2-(2,2-diphenyl)-2-prop-2-ynyloxy)acetoxy] ethylamine and its picrate and $(2 R, 3 R)$-(hydrogentartrate) salts (Shaibah et al., 2017).

\section{Synthesis and crystallization}

A sample of ebastine was a gift from RL Fine Chem, Pvt. Ltd., Bengaluru, India. For the synthesis of compound (I), ebastine $(100 \mathrm{mg}, 0.20 \mathrm{mmol})$ and 3,5-dinitrobenzoic acid $(45 \mathrm{mg}$, $0.20 \mathrm{mmol}$ ) were dissolved in hot methanol and held at $333 \mathrm{~K}$ for $30 \mathrm{~min}$, with magnetic stirring throughout. The resulting solution was then allowed to cool slowly to room temperature, giving colourless block-like crystals (m.p. 424-428 K).

\section{Refinement}

Crystal data, data collection and structure refinement details are summarized in Table 2. Three low-angle reflections (021), (002) and (012), which had been attenuated by the beam stop, were omitted from the refinements. It was apparent from an early stage in the refinement that the disubstituted aryl ring was disordered over two sets of atomic sights having unequal occupancies, and corresponding to different orientations of

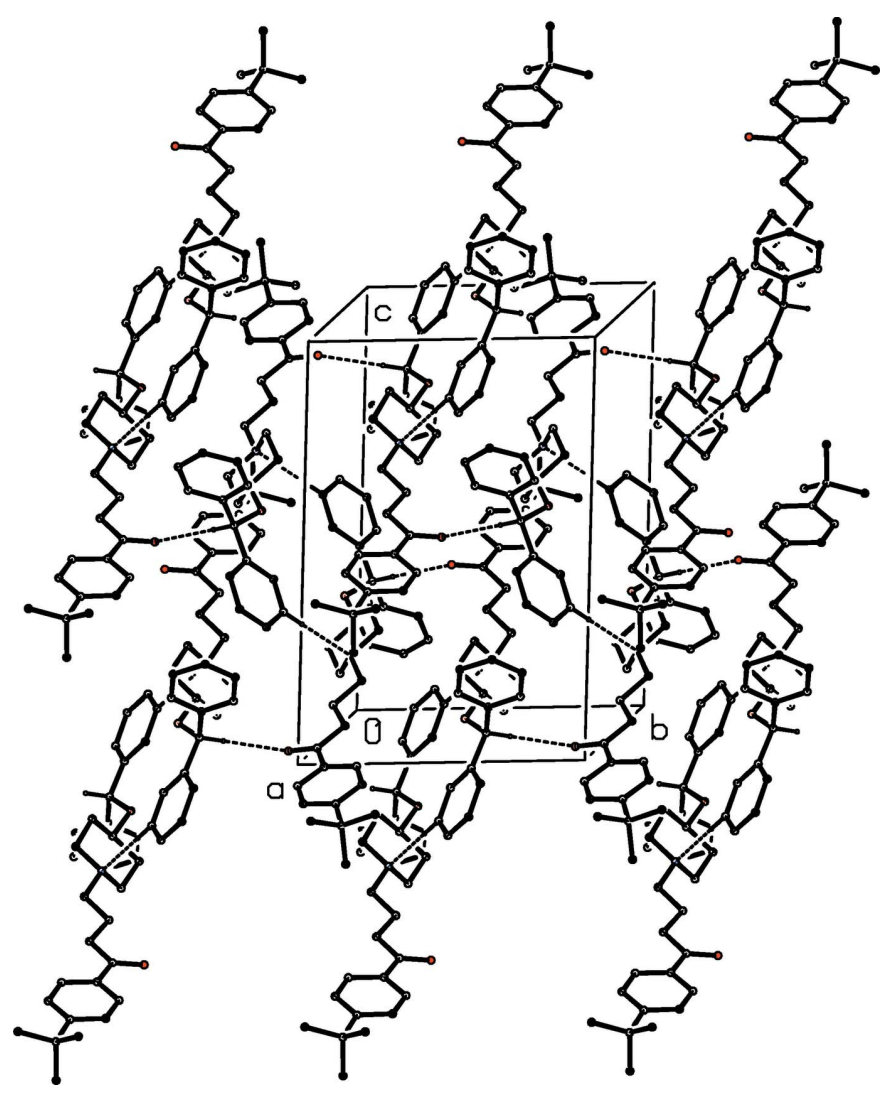

Figure 4

Part of the crystal structure of ebastine showing the formation of a hydrogen-bonded sheet of $R_{2}^{2}(20)$ and $R_{6}^{6}(48)$ rings. The original atomic coordinates (Sharma et al., 2015) have been used and, for the sake of clarity, the $\mathrm{H}$ atoms not involved in the motifs shown have been omitted. 
Table 2

Experimental details.

\begin{tabular}{|c|c|}
\hline \multicolumn{2}{|l|}{ Crystal data } \\
\hline Chemical formula & $\mathrm{C}_{32} \mathrm{H}_{40} \mathrm{NO}_{2} \cdot \mathrm{C}_{7} \mathrm{H}_{3} \mathrm{~N}_{2} \mathrm{O}_{6}$ \\
\hline$M_{\mathrm{r}}$ & 681.76 \\
\hline Crystal system, space group & Monoclinic, $P 2_{1} / c$ \\
\hline Temperature $(\mathrm{K})$ & 293 \\
\hline$a, b, c(\AA)$ & $\begin{array}{l}5.9168(3), 28.3733(12) \\
\quad 21.0782(11)\end{array}$ \\
\hline$\beta\left({ }^{\circ}\right)$ & $97.836(5)$ \\
\hline$V\left(\AA^{3}\right)$ & $3505.6(3)$ \\
\hline$Z$ & 4 \\
\hline Radiation type & Мо $K \alpha$ \\
\hline$\mu\left(\mathrm{mm}^{-1}\right)$ & 0.09 \\
\hline Crystal size $(\mathrm{mm})$ & $0.23 \times 0.21 \times 0.18$ \\
\hline \multicolumn{2}{|l|}{ Data collection } \\
\hline Diffractometer & Rigaku Saturn724 \\
\hline Absorption correction & $\begin{array}{l}\text { Multi-scan (SADABS; Krause et } \\
\quad \text { al., 2015) }\end{array}$ \\
\hline$T_{\min }, T_{\max }$ & $0.956,0.984$ \\
\hline $\begin{array}{l}\text { No. of measured, independent and } \\
\text { observed }[I>2 \sigma(I)] \text { reflections }\end{array}$ & $40112,7331,4388$ \\
\hline$R_{\text {int }}$ & 0.061 \\
\hline$(\sin \theta / \lambda)_{\max }\left(\AA^{-1}\right)$ & 0.629 \\
\hline \multicolumn{2}{|l|}{ Refinement } \\
\hline$R\left[F^{2}>2 \sigma\left(F^{2}\right)\right], w R\left(F^{2}\right), S$ & $0.065,0.179,1.05$ \\
\hline No. of reflections & 7331 \\
\hline No. of parameters & 470 \\
\hline No. of restraints & 22 \\
\hline $\mathrm{H}$-atom treatment & $\begin{array}{l}\mathrm{H} \text { atoms treated by a mixture of } \\
\text { independent and constrained } \\
\text { refinement }\end{array}$ \\
\hline$\Delta \rho_{\max }, \Delta \rho_{\min }\left(\mathrm{e} \AA^{-3}\right)$ & $0.20,-0.25$ \\
\hline
\end{tabular}

Computer programs: CrystalClear (Rigaku, 2011), SHELXS86 (Sheldrick, 2008), SHELXL2014 (Sheldrick, 2015) and PLATON (Spek, 2009).

this ring relative to its substituents. For the minor orientation, the bonded distances and the 1,3-non-bonded distances were restrained to be the same as the corresponding distances in the major orientation, subject to s.u.s of 0.01 and $0.02 \AA$, respectively: in addition, the anisotropic displacement parameters for corresponding pairs of atomic sites were constrained to be equal. All $\mathrm{H}$ atoms, other than those in the minor disorder components, were located in differenceFourier maps. The $\mathrm{C}$-bound $\mathrm{H}$ atoms were all treated as riding atoms in geometrically idealized positions: $\mathrm{C}-\mathrm{H} 0.93 \AA$ (aromatic), $0.96 \AA\left(\mathrm{CH}_{3}\right), 0.97 \AA\left(\mathrm{CH}_{2}\right)$ or $0.98 \AA$ (aliphatic $\mathrm{C}-\mathrm{H})$, with $U_{\text {iso }}(\mathrm{H})=1.5 U_{\text {eq }}(\mathrm{C}-$-methyl $)$ and $1.2 U_{\text {eq }}(\mathrm{C})$ for other $\mathrm{H}$ atoms. The methyl groups were permitted to rotate but not to tilt. For the $\mathrm{H}$ atom bonded to the $\mathrm{N}$ atom, the atomic coordinates were refined with $U_{\text {iso }}(\mathrm{H})=1.2 U_{\text {eq }}(\mathrm{N})$, giving an $\mathrm{N}-\mathrm{H}$ distance of 0.99 (3) $\AA$. Subject to these conditions, the occupancies of the two disordered components refined to $0.706(4)$ and $0.294(4)$. In the final analysis of variance there was a large value, 15.256, of $K=\left[\operatorname{mean}\left(F_{\mathrm{o}}^{2}\right) /\right.$ mean $\left.\left(F_{\mathrm{c}}^{2}\right)\right]$ for the group of 867 very weak reflections having $F_{\mathrm{c}} / F_{\mathrm{c}}(\max )$ in the range $0.000<F_{\mathrm{c}} / F_{\mathrm{c}}(\max )<0.005$.

\section{Acknowledgements}

The authors thank the DST-PURSE Lab. (Mangalore University) for the diffractometer and other facilities. MAES thanks the University of Mysore for research facilities and BKS thanks the UGC for the award of a Rajiv Gandhi National Fellowship.

\section{References}

Boeyens, J. C. A. (1978). J. Cryst. Mol. Struct. 8, 317-320.

Cheng, J., Zhou, Z. \& Yang, G. (2005). Acta Cryst. E61, o2932-o2933.

Ferguson, G., Glidewell, C., Gregson, R. M. \& Meehan, P. R. (1998a). Acta Cryst. B54, 129-138.

Ferguson, G., Glidewell, C., Gregson, R. M. \& Meehan, P. R. (1998b). Acta Cryst. B54, 139-150.

Gregson, R. M., Glidewell, C., Ferguson, G. \& Lough, A. J. (2000). Acta Cryst. B56, 39-57.

Jasinski, J. P., Butcher, R. J., Hakim AL-arique, Q. N. M., Yathirajan, H. S. \& Narayana, B. (2009). Acta Cryst. E65, o1738-o1739.

Krause, L., Herbst-Irmer, R., Sheldrick, G. M. \& Stalke, D. (2015). J. Appl. Cryst. 48, 3-10.

Rigaku (2011). CrystalClear. Rigaku Corporation, Tokyo, Japan.

Shaibah, M. A. E., Yathirajan, H. S., Kumar, S. M., Byrappa, K. \& Glidewell, C. (2017). E73, 1488-1493.

Sharma, R., Prasher, D. \& Tiwari, R. K. (2015). J. Appl. Cryst. 48, 1299-1301.

Sheldrick, G. M. (2008). Acta Cryst. A64, 112-122.

Sheldrick, G. M. (2015). Acta Cryst. C71, 3-8.

Spek, A. L. (2009). Acta Cryst. D65, 148-155.

Van Cauwenberge, P., De Belder, T. \& Sys, L. (2004). Expert Opin. Pharmacother. 5, 1807-1813.

Wiseman, L. R. \& Faulds, D. (1996). Drugs, 51, 260-277. 


\section{supporting information}

Acta Cryst. (2017). E73, 1513-1516 [https://doi.org/10.1107/S205698901701324X]

\section{Crystal structure of ebastinium 3,5-dinitrobenzoate}

Mohammed A. E. Shaibah, Belakavadi K. Sagar, Hemmige S. Yathirajan, S. Madan Kumar and

\section{Christopher Glidewell}

\section{Computing details}

Data collection: CrystalClear (Rigaku, 2011); cell refinement: CrystalClear (Rigaku, 2011); data reduction: CrystalClear (Rigaku, 2011); program(s) used to solve structure: SHELXS86 (Sheldrick, 2008); program(s) used to refine structure: SHELXL2014 (Sheldrick, 2015); molecular graphics: PLATON (Spek, 2009); software used to prepare material for publication: SHELXL2014 (Sheldrick, 2015) and PLATON (Spek, 2009).

4-(Benzhydryloxy)-1-[4-(4-tert-butylphenyl)-4-oxobutyl]piperidinium 3,5-dinitrobenzoate

Crystal data

$\mathrm{C}_{32} \mathrm{H}_{40} \mathrm{NO}_{2}{ }^{+} \cdot \mathrm{C}_{7} \mathrm{H}_{3} \mathrm{~N}_{2} \mathrm{O}_{6}{ }^{-}$

$M_{r}=681.76$

Monoclinic, $P 2_{1} / c$

$a=5.9168(3) \AA$

$b=28.3733(12) \AA$

$c=21.0782(11) \AA$

$\beta=97.836(5)^{\circ}$

$V=3505.6(3) \AA^{3}$

$Z=4$

Data collection

Rigaku Saturn724

diffractometer

Radiation source: fine focus sealed tube $\varphi$ and $\omega$ scans

Absorption correction: multi-scan

(SADABS; Krause et al., 2015)

$T_{\min }=0.956, T_{\max }=0.984$

40112 measured reflections

Refinement

Refinement on $F^{2}$

Least-squares matrix: full

$R\left[F^{2}>2 \sigma\left(F^{2}\right)\right]=0.065$

$w R\left(F^{2}\right)=0.179$

$S=1.05$

7331 reflections

470 parameters

22 restraints

Primary atom site location: structure-invariant direct methods
$F(000)=1448$

$D_{\mathrm{x}}=1.292 \mathrm{Mg} \mathrm{m}^{-3}$

Mo $K \alpha$ radiation, $\lambda=0.71073 \AA$

Cell parameters from 10431 reflections

$\theta=2.4-31.2^{\circ}$

$\mu=0.09 \mathrm{~mm}^{-1}$

$T=293 \mathrm{~K}$

Block, colourless

$0.23 \times 0.21 \times 0.18 \mathrm{~mm}$

7331 independent reflections 4388 reflections with $I>2 \sigma(I)$

$R_{\text {int }}=0.061$

$\theta_{\max }=26.6^{\circ}, \theta_{\min }=2.4^{\circ}$

$h=-7 \rightarrow 7$

$k=-35 \rightarrow 35$

$l=-26 \rightarrow 25$

Secondary atom site location: difference Fourier map

Hydrogen site location: mixed

$\mathrm{H}$ atoms treated by a mixture of independent and constrained refinement

$w=1 /\left[\sigma^{2}\left(F_{\mathrm{o}}^{2}\right)+(0.0661 P)^{2}+1.0412 P\right]$

where $P=\left(F_{\mathrm{o}}^{2}+2 F_{\mathrm{c}}^{2}\right) / 3$

$(\Delta / \sigma)_{\max }=0.001$

$\Delta \rho_{\max }=0.20 \mathrm{e} \AA^{-3}$

$\Delta \rho_{\min }=-0.25$ e $\AA^{-3}$ 


\section{Special details}

Geometry. All esds (except the esd in the dihedral angle between two 1.s. planes) are estimated using the full covariance matrix. The cell esds are taken into account individually in the estimation of esds in distances, angles and torsion angles; correlations between esds in cell parameters are only used when they are defined by crystal symmetry. An approximate (isotropic) treatment of cell esds is used for estimating esds involving l.s. planes.

Fractional atomic coordinates and isotropic or equivalent isotropic displacement parameters $\left(\AA^{2}\right)$

\begin{tabular}{|c|c|c|c|c|c|}
\hline & $x$ & $y$ & $z$ & $U_{\text {iso }} * / U_{\text {eq }}$ & Occ. $(<1)$ \\
\hline N1 & $0.3135(4)$ & $0.72898(6)$ & $0.37455(11)$ & $0.0510(5)$ & \\
\hline $\mathrm{H} 1$ & $0.374(4)$ & $0.7192(8)$ & $0.3349(12)$ & $0.061^{*}$ & \\
\hline $\mathrm{C} 2$ & $0.4460(4)$ & $0.77114(8)$ & $0.40021(11)$ & $0.0479(6)$ & \\
\hline $\mathrm{H} 2 \mathrm{~B}$ & 0.6062 & 0.7629 & 0.4097 & $0.057^{*}$ & \\
\hline $\mathrm{H} 2 \mathrm{~A}$ & 0.3938 & 0.7811 & 0.4398 & $0.057^{*}$ & \\
\hline C3 & 0.4185 (4) & $0.81101(8)$ & $0.35285(11)$ & $0.0457(6)$ & \\
\hline $\mathrm{H} 3 \mathrm{~A}$ & 0.4826 & 0.8018 & 0.3147 & $0.055^{*}$ & \\
\hline H3B & 0.5027 & 0.8382 & 0.3713 & $0.055^{*}$ & \\
\hline $\mathrm{C} 4$ & $0.1701(4)$ & $0.82448(8)$ & $0.33437(11)$ & $0.0457(6)$ & \\
\hline $\mathrm{H} 4$ & 0.1579 & 0.8488 & 0.3011 & $0.055^{*}$ & \\
\hline $\mathrm{C} 5$ & $0.0386(4)$ & $0.78137(9)$ & $0.30906(12)$ & $0.0553(7)$ & \\
\hline $\mathrm{H} 5 \mathrm{~A}$ & -0.1219 & 0.7892 & 0.2994 & $0.066^{*}$ & \\
\hline H5B & 0.0916 & 0.7713 & 0.2697 & $0.066^{*}$ & \\
\hline C6 & $0.0676(4)$ & $0.74172(9)$ & $0.35682(13)$ & $0.0575(7)$ & \\
\hline H6A & 0.0043 & 0.7510 & 0.3950 & $0.069^{*}$ & \\
\hline H6B & -0.0160 & 0.7144 & 0.3388 & $0.069^{*}$ & \\
\hline $\mathrm{O} 4$ & $0.0623(3)$ & $0.84055(5)$ & $0.38735(7)$ & $0.0484(4)$ & \\
\hline $\mathrm{C} 41$ & 0.1566 (4) & $0.88157(8)$ & $0.41962(11)$ & $0.0460(6)$ & \\
\hline $\mathrm{H} 41$ & 0.3107 & 0.8739 & 0.4403 & $0.055^{*}$ & \\
\hline C141 & 0.0098 (4) & $0.89282(8)$ & $0.47183(11)$ & $0.0453(6)$ & \\
\hline C142 & $-0.1660(4)$ & $0.86412(9)$ & 0.48464 (11) & $0.0511(6)$ & \\
\hline H142 & -0.1975 & 0.8367 & 0.4610 & $0.061^{*}$ & \\
\hline C143 & $-0.2974(5)$ & $0.87546(10)$ & $0.53237(12)$ & $0.0613(7)$ & \\
\hline H143 & -0.4159 & 0.8557 & 0.5403 & $0.074 *$ & \\
\hline C144 & $-0.2527(6)$ & $0.91565(10)$ & $0.56772(13)$ & $0.0686(8)$ & \\
\hline H144 & -0.3422 & 0.9236 & 0.5991 & $0.082 *$ & \\
\hline C145 & $-0.0755(6)$ & $0.94408(10)$ & $0.55665(15)$ & $0.0798(10)$ & \\
\hline H145 & -0.0426 & 0.9711 & 0.5812 & $0.096^{*}$ & \\
\hline C146 & $0.0550(6)$ & $0.93287(9)$ & 0.50904 (14) & $0.0719(8)$ & \\
\hline H146 & 0.1749 & 0.9525 & 0.5019 & $0.086^{*}$ & \\
\hline $\mathrm{C} 151$ & 0.1735 (4) & $0.92244(8)$ & 0.37464 (11) & $0.0469(6)$ & \\
\hline C152 & $0.3761(5)$ & $0.94689(9)$ & $0.37596(14)$ & $0.0612(7)$ & \\
\hline H152 & 0.5016 & 0.9380 & 0.4050 & $0.073^{*}$ & \\
\hline $\mathrm{C} 153$ & $0.3962(6)$ & $0.98416(10)$ & $0.33510(18)$ & $0.0770(9)$ & \\
\hline H153 & 0.5338 & 1.0003 & 0.3368 & $0.092 *$ & \\
\hline $\mathrm{C} 154$ & $0.2134(7)$ & $0.99730(11)$ & $0.29230(17)$ & $0.0808(10)$ & \\
\hline H154 & 0.2264 & 1.0222 & 0.2644 & $0.097^{*}$ & \\
\hline C155 & $0.0086(6)$ & $0.97356(11)$ & $0.29040(15)$ & $0.0754(9)$ & \\
\hline H155 & -0.1161 & 0.9827 & 0.2613 & $0.091^{*}$ & \\
\hline
\end{tabular}




\begin{tabular}{|c|c|c|c|c|c|}
\hline C156 & $-0.0118(5)$ & $0.93637(9)$ & $0.33152(13)$ & $0.0603(7)$ & \\
\hline H156 & -0.1504 & 0.9207 & 0.3302 & $0.072 *$ & \\
\hline C11 & $0.3376(6)$ & $0.68892(10)$ & $0.42095(17)$ & $0.0865(11)$ & \\
\hline H11A & 0.2213 & 0.6656 & 0.4071 & $0.104^{*}$ & \\
\hline H11B & 0.3101 & 0.7005 & 0.4625 & $0.104 *$ & \\
\hline C12 & $0.5687(5)$ & $0.66527(9)$ & $0.42801(16)$ & $0.0724(9)$ & \\
\hline $\mathrm{H} 12 \mathrm{~A}$ & 0.6523 & 0.6664 & 0.4737 & $0.087^{*}$ & \\
\hline H12B & 0.6523 & 0.6776 & 0.4014 & $0.087^{*}$ & \\
\hline C13 & $0.5462(5)$ & $0.61430(10)$ & $0.41343(17)$ & $0.0833(10)$ & \\
\hline H13A & 0.4697 & 0.6108 & 0.3700 & $0.100 *$ & \\
\hline H13B & 0.4481 & 0.6005 & 0.4417 & $0.100^{*}$ & \\
\hline C14 & $0.7637(6)$ & $0.58644(10)$ & $0.41954(15)$ & $0.0709(8)$ & \\
\hline $\mathrm{O} 14$ & $0.9451(4)$ & $0.60527(8)$ & $0.43408(16)$ & $0.1170(10)$ & \\
\hline C161 & $0.7488(5)$ & $0.53513(9)$ & $0.40590(14)$ & $0.0659(8)$ & $0.706(4)$ \\
\hline C162 & $0.5497(7)$ & $0.51590(14)$ & $0.3727(3)$ & 0.0902 (17) & $0.706(4)$ \\
\hline H162 & 0.4245 & 0.5351 & 0.3599 & $0.108^{*}$ & $0.706(4)$ \\
\hline C163 & $0.5386(7)$ & $0.46828(14)$ & $0.3589(3)$ & 0.0907 (18) & $0.706(4)$ \\
\hline H163 & 0.4054 & 0.4561 & 0.3364 & $0.109^{*}$ & $0.706(4)$ \\
\hline C164 & $0.7209(5)$ & $0.43792(9)$ & $0.37782(13)$ & $0.0587(7)$ & $0.706(4)$ \\
\hline $\mathrm{C} 165$ & $0.9157(9)$ & $0.45915(18)$ & $0.4048(5)$ & $0.103(4)$ & $0.706(4)$ \\
\hline H165 & 1.0458 & 0.4407 & 0.4147 & $0.124^{*}$ & $0.706(4)$ \\
\hline C166 & $0.9308(9)$ & $0.50664(17)$ & $0.4185(4)$ & $0.099(3)$ & $0.706(4)$ \\
\hline H166 & 1.0697 & 0.5192 & 0.4367 & $0.119^{*}$ & $0.706(4)$ \\
\hline C171 & $0.7488(5)$ & $0.53513(9)$ & $0.40590(14)$ & $0.0659(8)$ & $0.294(4)$ \\
\hline $\mathrm{C} 172$ & $0.5662(15)$ & $0.5073(3)$ & $0.4211(5)$ & $0.0902(17)$ & $0.294(4)$ \\
\hline H172 & 0.4497 & 0.5216 & 0.4396 & $0.108^{*}$ & $0.294(4)$ \\
\hline $\mathrm{C} 173$ & $0.5554(15)$ & $0.4594(3)$ & $0.4093(5)$ & 0.0907 (18) & $0.294(4)$ \\
\hline H173 & 0.4382 & 0.4415 & 0.4223 & $0.109 *$ & $0.294(4)$ \\
\hline $\mathrm{C} 174$ & $0.7209(5)$ & $0.43792(9)$ & $0.37782(13)$ & $0.0587(7)$ & $0.294(4)$ \\
\hline $\mathrm{C} 175$ & $0.9160(17)$ & $0.4626(4)$ & $0.3793(15)$ & $0.103(4)$ & $0.294(4)$ \\
\hline H175 & 1.0483 & 0.4468 & 0.3726 & $0.124^{*}$ & 0.294 (4) \\
\hline $\mathrm{C} 176$ & $0.9267(18)$ & $0.5104(4)$ & 0.3905 (12) & 0.099 (3) & $0.294(4)$ \\
\hline H176 & 1.0625 & 0.5262 & 0.3872 & $0.119^{*}$ & $0.294(4)$ \\
\hline C181 & $0.7009(5)$ & $0.38550(9)$ & $0.36135(13)$ & $0.0591(7)$ & \\
\hline C182 & $0.6091(6)$ & $0.35909(12)$ & $0.41549(16)$ & $0.0925(11)$ & \\
\hline H18A & 0.5908 & 0.3264 & 0.4044 & $0.139^{*}$ & \\
\hline H18B & 0.4643 & 0.3721 & 0.4220 & $0.139 *$ & \\
\hline $\mathrm{H} 18 \mathrm{C}$ & 0.7145 & 0.3622 & 0.4541 & $0.139^{*}$ & \\
\hline C183 & $0.9278(5)$ & $0.36413(11)$ & $0.35081(18)$ & $0.0903(11)$ & \\
\hline H18D & 1.0326 & 0.3661 & 0.3897 & $0.135^{*}$ & \\
\hline $\mathrm{H} 18 \mathrm{E}$ & 0.9883 & 0.3811 & 0.3175 & $0.135 *$ & \\
\hline $\mathrm{H} 18 \mathrm{~F}$ & 0.9059 & 0.3317 & 0.3386 & $0.135^{*}$ & \\
\hline C184 & $0.5349(5)$ & $0.37825(11)$ & $0.29982(14)$ & $0.0753(8)$ & \\
\hline $\mathrm{H} 18 \mathrm{G}$ & 0.5895 & 0.3949 & 0.2653 & $0.113^{*}$ & \\
\hline $\mathrm{H} 18 \mathrm{H}$ & 0.3872 & 0.3900 & 0.3058 & $0.113^{*}$ & \\
\hline H18I & 0.5240 & 0.3453 & 0.2898 & $0.113^{*}$ & \\
\hline $\mathrm{C} 21$ & $0.7522(4)$ & $0.69940(8)$ & $0.19856(11)$ & $0.0431(5)$ & \\
\hline $\mathrm{C} 22$ & $0.8763(4)$ & $0.66079(8)$ & $0.22368(11)$ & $0.0458(6)$ & \\
\hline
\end{tabular}




$\begin{array}{lllll}\mathrm{H} 22 & 0.8338 & 0.6448 & 0.2587 & 0.055^{*} \\ \mathrm{C} 23 & 1.0631(4) & 0.64614(8) & 0.19644(12) & 0.0486(6) \\ \mathrm{C} 24 & 1.1306(4) & 0.66812(9) & 0.14419(12) & 0.0525(6) \\ \mathrm{H} 24 & 1.2571 & 0.6579 & 0.1263 & 0.063^{*} \\ \mathrm{C} 25 & 1.0032(4) & 0.70587(9) & 0.11964(11) & 0.0505(6) \\ \mathrm{C} 26 & 0.8156(4) & 0.72192(8) & 0.14567(11) & 0.0493(6) \\ \mathrm{H} 26 & 0.7327 & 0.7476 & 0.1278 & 0.059^{*} \\ \mathrm{C} 27 & 0.5535(4) & 0.71822(9) & 0.23013(13) & 0.0504(6) \\ \mathrm{O} 21 & 0.4514(3) & 0.75292(7) & 0.20644(9) & 0.0689(5) \\ \mathrm{O} 22 & 0.5158(3) & 0.69618(6) & 0.27979(9) & 0.0649(5) \\ \mathrm{N} 23 & 1.1983(4) & 0.60593(8) & 0.22480(13) & 0.0672(6) \\ \mathrm{O} 23 & 1.1460(4) & 0.58894(8) & 0.27369(12) & 0.0920(7) \\ \mathrm{O} 24 & 1.3561(4) & 0.59235(8) & 0.19822(12) & 0.1010(8) \\ \mathrm{N} 25 & 1.0724(5) & 0.72964(9) & 0.06325(12) & 0.0700(7) \\ \mathrm{O} 25 & 1.2546(4) & 0.71894(9) & 0.04679(11) & 0.0974(8) \\ \mathrm{O} 26 & 0.9409(5) & 0.75808(9) & 0.03539(11) & 0.1034(8)\end{array}$

Atomic displacement parameters $\left(\AA^{2}\right)$

\begin{tabular}{|c|c|c|c|c|c|c|}
\hline & $U^{11}$ & $U^{22}$ & $U^{33}$ & $U^{12}$ & $U^{13}$ & $U^{23}$ \\
\hline N1 & 0.0585 (13) & $0.0405(10)$ & $0.0606(13)$ & $0.0002(9)$ & $0.0321(11)$ & $-0.0031(10)$ \\
\hline $\mathrm{C} 2$ & $0.0490(14)$ & 0.0496 (13) & $0.0474(14)$ & 0.0035 (11) & $0.0147(11)$ & $-0.0072(11)$ \\
\hline C3 & $0.0470(14)$ & $0.0421(12)$ & 0.0508 (14) & $-0.0017(10)$ & $0.0168(11)$ & $-0.0075(11)$ \\
\hline $\mathrm{C} 4$ & $0.0480(14)$ & $0.0472(13)$ & $0.0444(13)$ & $0.0039(11)$ & $0.0150(11)$ & $-0.0043(11)$ \\
\hline $\mathrm{C} 5$ & $0.0430(14)$ & $0.0664(16)$ & $0.0576(16)$ & 0.0005 (12) & 0.0108 (12) & $-0.0223(13)$ \\
\hline C6 & $0.0514(16)$ & $0.0545(15)$ & $0.0722(18)$ & $-0.0128(12)$ & $0.0285(14)$ & $-0.0229(14)$ \\
\hline $\mathrm{O} 4$ & $0.0520(10)$ & $0.0442(9)$ & $0.0527(10)$ & $0.0011(7)$ & $0.0203(8)$ & $-0.0131(7)$ \\
\hline C41 & $0.0446(13)$ & $0.0415(12)$ & $0.0521(14)$ & $0.0040(10)$ & $0.0076(11)$ & $-0.0054(11)$ \\
\hline C141 & 0.0513 (14) & $0.0397(12)$ & $0.0453(13)$ & $0.0080(11)$ & $0.0082(11)$ & $-0.0019(10)$ \\
\hline C142 & $0.0510(15)$ & $0.0579(15)$ & $0.0441(14)$ & $0.0011(12)$ & $0.0058(12)$ & $-0.0045(12)$ \\
\hline C143 & $0.0581(17)$ & 0.0772 (19) & $0.0501(15)$ & 0.0059 (14) & $0.0130(13)$ & $0.0065(14)$ \\
\hline C144 & $0.088(2)$ & $0.0690(18)$ & $0.0540(17)$ & $0.0236(17)$ & $0.0277(16)$ & $0.0054(15)$ \\
\hline $\mathrm{C} 145$ & $0.122(3)$ & $0.0537(17)$ & $0.070(2)$ & $0.0072(18)$ & $0.040(2)$ & $-0.0174(15)$ \\
\hline $\mathrm{C} 146$ & $0.100(2)$ & $0.0518(16)$ & 0.0696 (19) & $-0.0091(15)$ & $0.0334(18)$ & $-0.0129(14)$ \\
\hline $\mathrm{C} 151$ & 0.0498 (14) & $0.0416(12)$ & $0.0521(14)$ & $0.0045(11)$ & $0.0171(12)$ & $-0.0075(11)$ \\
\hline C152 & 0.0595 (17) & $0.0526(15)$ & 0.0755 (19) & 0.0007 (13) & $0.0234(15)$ & $-0.0081(14)$ \\
\hline C153 & $0.079(2)$ & 0.0557 (17) & $0.106(3)$ & $0.0001(16)$ & $0.047(2)$ & $-0.0010(18)$ \\
\hline C154 & $0.117(3)$ & $0.0544(17)$ & $0.083(2)$ & 0.0105 (19) & $0.056(2)$ & $0.0096(16)$ \\
\hline C155 & $0.092(2)$ & 0.0709 (19) & 0.0646 (19) & $0.0251(18)$ & 0.0133 (17) & $0.0088(16)$ \\
\hline C156 & $0.0614(17)$ & $0.0567(16)$ & $0.0640(17)$ & $0.0072(13)$ & $0.0131(14)$ & $0.0013(14)$ \\
\hline C11 & $0.109(3)$ & $0.0547(16)$ & 0.110 & $0.0158(17)$ & $0.069(2)$ & $0.0279(17)$ \\
\hline $\mathrm{C} 12$ & $0.089(2)$ & $0.0500(15)$ & $0.086(2)$ & $0.0124(15)$ & $0.0381(18)$ & $0.0181(15)$ \\
\hline $\mathrm{C} 13$ & $0.087(2)$ & 0.0635 (18) & $0.095(2)$ & $0.0207(16)$ & $-0.0026(19)$ & $-0.0269(17)$ \\
\hline $\mathrm{C} 14$ & $0.073(2)$ & $0.0543(16)$ & $0.083(2)$ & $0.0038(15)$ & $0.0015(17)$ & $-0.0062(15)$ \\
\hline O14 & $0.0782(17)$ & $0.0650(14)$ & $0.200(3)$ & $-0.0039(12)$ & $-0.0078(17)$ & $-0.0152(16)$ \\
\hline C161 & $0.0586(17)$ & $0.0523(15)$ & $0.085(2)$ & $0.0033(14)$ & $0.0038(15)$ & $-0.0064(14)$ \\
\hline C162 & $0.071(3)$ & $0.063(2)$ & $0.125(5)$ & $0.028(2)$ & -0.031 & $-0.023(3)$ \\
\hline C163 & $0.062(2)$ & $0.065(2)$ & $0.136(5)$ & 0.0107 (19) & -0.023 & $-0.029(3)$ \\
\hline
\end{tabular}




$\begin{array}{lllllll}\text { C164 } & 0.0531(16) & 0.0537(15) & 0.0714(18) & 0.0057(13) & 0.0157(14) & -0.0063(13) \\ \text { C165 } & 0.064(2) & 0.056(2) & 0.181(11) & 0.0166(17) & -0.013(3) & -0.014(4) \\ \text { C166 } & 0.061(2) & 0.057(2) & 0.170(10) & 0.0066(17) & -0.017(3) & -0.011(3) \\ \text { C171 } & 0.0586(17) & 0.0523(15) & 0.085(2) & 0.0033(14) & 0.0038(15) & -0.0064(14) \\ \text { C172 } & 0.071(3) & 0.063(2) & 0.125(5) & 0.028(2) & -0.031(3) & -0.023(3) \\ \text { C173 } & 0.062(2) & 0.065(2) & 0.136(5) & 0.0107(19) & -0.023(3) & -0.029(3) \\ \text { C174 } & 0.0531(16) & 0.0537(15) & 0.0714(18) & 0.0057(13) & 0.0157(14) & -0.0063(13) \\ \text { C175 } & 0.064(2) & 0.056(2) & 0.181(11) & 0.0166(17) & -0.013(3) & -0.014(4) \\ \text { C176 } & 0.061(2) & 0.057(2) & 0.170(10) & 0.0066(17) & -0.017(3) & -0.011(3) \\ \text { C181 } & 0.0610(17) & 0.0529(15) & 0.0659(17) & 0.0014(13) & 0.0175(14) & 0.0020(13) \\ \text { C182 } & 0.121(3) & 0.087(2) & 0.073(2) & -0.007(2) & 0.028(2) & 0.0090(18) \\ \text { C183 } & 0.079(2) & 0.069(2) & 0.124(3) & 0.0164(17) & 0.019(2) & -0.014(2) \\ \text { C184 } & 0.085(2) & 0.0685(18) & 0.074(2) & -0.0021(16) & 0.0180(17) & -0.0076(16) \\ \text { C21 } & 0.0455(13) & 0.0423(12) & 0.0432(13) & -0.0045(10) & 0.0127(11) & -0.0105(10) \\ \text { C22 } & 0.0518(14) & 0.0467(13) & 0.0406(13) & -0.0047(11) & 0.0124(11) & -0.0067(10) \\ \text { C23 } & 0.0479(14) & 0.0481(13) & 0.0496(14) & 0.0050(11) & 0.0062(12) & -0.0117(11) \\ \text { C24 } & 0.0476(14) & 0.0601(15) & 0.0527(15) & -0.0056(12) & 0.0173(12) & -0.0186(13) \\ \text { C25 } & 0.0535(15) & 0.0581(15) & 0.0428(14) & -0.0097(12) & 0.0169(12) & -0.0076(12) \\ \text { C26 } & 0.0536(15) & 0.0480(13) & 0.0472(14) & -0.0034(11) & 0.0106(12) & -0.0072(11) \\ \text { C27 } & 0.0486(15) & 0.0505(14) & 0.0548(16) & -0.0031(12) & 0.0166(12) & -0.0165(13) \\ \text { O21 } & 0.0677(13) & 0.0620(12) & 0.0798(13) & 0.0171(10) & 0.0207(10) & -0.0063(10) \\ \text { O22 } & 0.0747(13) & 0.0646(11) & 0.0634(12) & 0.0063(9) & 0.0386(10) & -0.0071(10) \\ \text { N23 } & 0.0668(16) & 0.0624(15) & 0.0715(16) & 0.0143(12) & 0.0064(14) & -0.0117(13) \\ \text { O23 } & 0.1038(18) & 0.0849(15) & 0.0873(16) & 0.0259(13) & 0.0133(14) & 0.0247(13) \\ \text { O24 } & 0.0920(17) & 0.1013(17) & 0.1138(19) & 0.0466(14) & 0.0293(15) & -0.0112(15) \\ \text { N25 } & 0.0849(19) & 0.0748(17) & 0.0557(15) & -0.0129(14) & 0.0292(15) & -0.0015(13) \\ \text { O25 } & 0.0960(18) & 0.127(2) & 0.0806(16) & -0.0088(15) & 0.0543(14) & -0.0002(14) \\ \text { O26 } & 0.132(2) & 0.1008(18) & 0.0844(17) & 0.0167(16) & 0.0413(16) & 0.0340(15) \\ & & & & & \end{array}$

Geometric parameters ( $\left.\AA,{ }^{\circ}\right)$

\begin{tabular}{llll}
\hline $\mathrm{N} 1-\mathrm{C} 2$ & $1.491(3)$ & $\mathrm{C} 13-\mathrm{H} 13 \mathrm{~A}$ & 0.9700 \\
$\mathrm{~N} 1-\mathrm{C} 11$ & $1.493(3)$ & $\mathrm{C} 13-\mathrm{H} 13 \mathrm{~B}$ & 0.9700 \\
$\mathrm{~N} 1-\mathrm{C} 6$ & $1.496(3)$ & $\mathrm{C} 14-\mathrm{O} 14$ & $1.201(3)$ \\
$\mathrm{N} 1-\mathrm{H} 1$ & $0.99(3)$ & $\mathrm{C} 14-\mathrm{C} 161$ & $1.344(5)$ \\
$\mathrm{C} 2-\mathrm{C} 3$ & $1.503(3)$ & $\mathrm{C} 161-\mathrm{C} 166$ & $1.397(5)$ \\
$\mathrm{C} 2-\mathrm{H} 2 \mathrm{~B}$ & 0.9700 & $\mathrm{C} 161-\mathrm{C} 162$ & $1.382(5)$ \\
$\mathrm{C} 2-\mathrm{H} 2 \mathrm{~A}$ & 0.9700 & $\mathrm{C} 162-\mathrm{C} 163$ & 0.9300 \\
$\mathrm{C} 3-\mathrm{C} 4$ & $1.517(3)$ & $\mathrm{C} 162-\mathrm{H} 162$ & $1.396(4)$ \\
$\mathrm{C} 3-\mathrm{H} 3 \mathrm{~A}$ & 0.9700 & $\mathrm{C} 163-\mathrm{C} 164$ & 0.9300 \\
$\mathrm{C} 3-\mathrm{H} 3 \mathrm{~B}$ & 0.9700 & $\mathrm{C} 163-\mathrm{H} 163$ & $1.355(6)$ \\
$\mathrm{C} 4-\mathrm{O} 4$ & $1.434(3)$ & $\mathrm{C} 164-\mathrm{C} 165$ & $1.528(4)$ \\
$\mathrm{C} 4-\mathrm{C} 5$ & $1.507(3)$ & $\mathrm{C} 164-\mathrm{C} 181$ & $1.378(5)$ \\
$\mathrm{C} 4-\mathrm{H} 4$ & 0.9800 & $\mathrm{C} 165-\mathrm{C} 166$ & 0.9300 \\
$\mathrm{C} 5-\mathrm{C} 6$ & $1.504(4)$ & $\mathrm{C} 165-\mathrm{H} 165$ & 0.9300 \\
$\mathrm{C} 5-\mathrm{H} 5 \mathrm{~A}$ & 0.9700 & $\mathrm{C} 166-\mathrm{H} 166$ & $1.381(8)$ \\
$\mathrm{C} 5-\mathrm{H} 5 \mathrm{~B}$ & 0.9700 & $\mathrm{C} 172-\mathrm{C} 173$ & 0.9300 \\
$\mathrm{C} 6-\mathrm{H} 6 \mathrm{~A}$ & 0.9700 & $\mathrm{C} 172-\mathrm{H} 172$ &
\end{tabular}




\begin{tabular}{|c|c|c|c|}
\hline $\mathrm{C} 6-\mathrm{H} 6 \mathrm{~B}$ & 0.9700 & C173-H173 & 0.9300 \\
\hline $\mathrm{O} 4-\mathrm{C} 41$ & $1.423(3)$ & $\mathrm{C} 175-\mathrm{C} 176$ & $1.376(8)$ \\
\hline $\mathrm{C} 41-\mathrm{C} 151$ & $1.510(3)$ & $\mathrm{C} 175-\mathrm{H} 175$ & 0.9300 \\
\hline $\mathrm{C} 41-\mathrm{C} 141$ & $1.526(3)$ & $\mathrm{C} 176-\mathrm{H} 176$ & 0.9300 \\
\hline $\mathrm{C} 41-\mathrm{H} 41$ & 0.9800 & $\mathrm{C} 181-\mathrm{C} 183$ & $1.517(4)$ \\
\hline $\mathrm{C} 141-\mathrm{C} 142$ & $1.377(3)$ & $\mathrm{C} 181-\mathrm{C} 182$ & $1.526(4)$ \\
\hline $\mathrm{C} 141-\mathrm{C} 146$ & $1.386(3)$ & $\mathrm{C} 181-\mathrm{C} 184$ & $1.530(4)$ \\
\hline $\mathrm{C} 142-\mathrm{C} 143$ & $1.390(3)$ & $\mathrm{C} 182-\mathrm{H} 18 \mathrm{~A}$ & 0.9600 \\
\hline C142-H142 & 0.9300 & $\mathrm{C} 182-\mathrm{H} 18 \mathrm{~B}$ & 0.9600 \\
\hline C143-C144 & $1.368(4)$ & $\mathrm{C} 182-\mathrm{H} 18 \mathrm{C}$ & 0.9600 \\
\hline C143-H143 & 0.9300 & $\mathrm{C} 183-\mathrm{H} 18 \mathrm{D}$ & 0.9600 \\
\hline C144-C145 & $1.368(4)$ & $\mathrm{C} 183-\mathrm{H} 18 \mathrm{E}$ & 0.9600 \\
\hline C144-H144 & 0.9300 & $\mathrm{C} 183-\mathrm{H} 18 \mathrm{~F}$ & 0.9600 \\
\hline C145-C146 & $1.384(4)$ & $\mathrm{C} 184-\mathrm{H} 18 \mathrm{G}$ & 0.9600 \\
\hline C145-H145 & 0.9300 & $\mathrm{C} 184-\mathrm{H} 18 \mathrm{H}$ & 0.9600 \\
\hline $\mathrm{C} 146-\mathrm{H} 146$ & 0.9300 & C184-H18I & 0.9600 \\
\hline $\mathrm{C} 151-\mathrm{C} 152$ & $1.382(3)$ & $\mathrm{C} 21-\mathrm{C} 26$ & $1.380(3)$ \\
\hline $\mathrm{C} 151-\mathrm{C} 156$ & $1.383(4)$ & $\mathrm{C} 21-\mathrm{C} 22$ & $1.383(3)$ \\
\hline $\mathrm{C} 152-\mathrm{C} 153$ & $1.379(4)$ & $\mathrm{C} 21-\mathrm{C} 27$ & $1.524(3)$ \\
\hline $\mathrm{C} 152-\mathrm{H} 152$ & 0.9300 & $\mathrm{C} 22-\mathrm{C} 23$ & $1.378(3)$ \\
\hline $\mathrm{C} 153-\mathrm{C} 154$ & $1.363(5)$ & $\mathrm{C} 22-\mathrm{H} 22$ & 0.9300 \\
\hline C153-H153 & 0.9300 & $\mathrm{C} 23-\mathrm{C} 24$ & $1.371(3)$ \\
\hline $\mathrm{C} 154-\mathrm{C} 155$ & $1.382(5)$ & $\mathrm{C} 23-\mathrm{N} 23$ & $1.473(3)$ \\
\hline C154-H154 & 0.9300 & $\mathrm{C} 24-\mathrm{C} 25$ & $1.370(3)$ \\
\hline $\mathrm{C} 155-\mathrm{C} 156$ & $1.381(4)$ & $\mathrm{C} 24-\mathrm{H} 24$ & 0.9300 \\
\hline $\mathrm{C} 155-\mathrm{H} 155$ & 0.9300 & $\mathrm{C} 25-\mathrm{C} 26$ & $1.381(3)$ \\
\hline $\mathrm{C} 156-\mathrm{H} 156$ & 0.9300 & $\mathrm{C} 25-\mathrm{N} 25$ & $1.472(3)$ \\
\hline $\mathrm{C} 11-\mathrm{C} 12$ & $1.512(4)$ & $\mathrm{C} 26-\mathrm{H} 26$ & 0.9300 \\
\hline $\mathrm{C} 11-\mathrm{H} 11 \mathrm{~A}$ & 0.9700 & $\mathrm{C} 27-\mathrm{O} 21$ & $1.226(3)$ \\
\hline $\mathrm{C} 11-\mathrm{H} 11 \mathrm{~B}$ & 0.9700 & $\mathrm{C} 27-\mathrm{O} 22$ & $1.265(3)$ \\
\hline $\mathrm{C} 12-\mathrm{C} 13$ & $1.481(4)$ & $\mathrm{N} 23-\mathrm{O} 24$ & $1.215(3)$ \\
\hline $\mathrm{C} 12-\mathrm{H} 12 \mathrm{~A}$ & 1.0216 & $\mathrm{~N} 23-\mathrm{O} 23$ & $1.215(3)$ \\
\hline $\mathrm{C} 12-\mathrm{H} 12 \mathrm{~B}$ & 0.8703 & $\mathrm{~N} 25-\mathrm{O} 26$ & $1.215(3)$ \\
\hline $\mathrm{C} 13-\mathrm{C} 14$ & $1.501(4)$ & $\mathrm{N} 25-\mathrm{O} 25$ & $1.215(3)$ \\
\hline $\mathrm{C} 2-\mathrm{N} 1-\mathrm{C} 11$ & $112.0(2)$ & $\mathrm{C} 11-\mathrm{C} 12-\mathrm{H} 12 \mathrm{~A}$ & 113.2 \\
\hline $\mathrm{C} 2-\mathrm{N} 1-\mathrm{C} 6$ & $110.00(18)$ & $\mathrm{C} 13-\mathrm{C} 12-\mathrm{H} 12 \mathrm{~B}$ & 107.6 \\
\hline $\mathrm{C} 11-\mathrm{N} 1-\mathrm{C} 6$ & $110.5(2)$ & $\mathrm{C} 11-\mathrm{C} 12-\mathrm{H} 12 \mathrm{~B}$ & 110.1 \\
\hline $\mathrm{C} 2-\mathrm{N} 1-\mathrm{H} 1$ & $107.3(14)$ & $\mathrm{H} 12 \mathrm{~A}-\mathrm{C} 12-\mathrm{H} 12 \mathrm{~B}$ & 110.5 \\
\hline $\mathrm{C} 11-\mathrm{N} 1-\mathrm{H} 1$ & $109.0(14)$ & $\mathrm{C} 12-\mathrm{C} 13-\mathrm{C} 14$ & $116.4(3)$ \\
\hline $\mathrm{C} 6-\mathrm{N} 1-\mathrm{H} 1$ & $107.9(15)$ & $\mathrm{C} 12-\mathrm{C} 13-\mathrm{H} 13 \mathrm{~A}$ & 108.2 \\
\hline $\mathrm{N} 1-\mathrm{C} 2-\mathrm{C} 3$ & $111.04(19)$ & $\mathrm{C} 14-\mathrm{C} 13-\mathrm{H} 13 \mathrm{~A}$ & 108.2 \\
\hline $\mathrm{N} 1-\mathrm{C} 2-\mathrm{H} 2 \mathrm{~B}$ & 109.4 & $\mathrm{C} 12-\mathrm{C} 13-\mathrm{H} 13 \mathrm{~B}$ & 108.2 \\
\hline $\mathrm{C} 3-\mathrm{C} 2-\mathrm{H} 2 \mathrm{~B}$ & 109.4 & $\mathrm{C} 14-\mathrm{C} 13-\mathrm{H} 13 \mathrm{~B}$ & 108.2 \\
\hline $\mathrm{N} 1-\mathrm{C} 2-\mathrm{H} 2 \mathrm{~A}$ & 109.4 & $\mathrm{H} 13 \mathrm{~A}-\mathrm{C} 13-\mathrm{H} 13 \mathrm{~B}$ & 107.3 \\
\hline $\mathrm{C} 3-\mathrm{C} 2-\mathrm{H} 2 \mathrm{~A}$ & 109.4 & $\mathrm{O} 14-\mathrm{C} 14-\mathrm{C} 161$ & $120.9(3)$ \\
\hline $\mathrm{H} 2 \mathrm{~B}-\mathrm{C} 2-\mathrm{H} 2 \mathrm{~A}$ & 108.0 & $\mathrm{O} 14-\mathrm{C} 14-\mathrm{C} 13$ & $120.9(3)$ \\
\hline $\mathrm{C} 2-\mathrm{C} 3-\mathrm{C} 4$ & $111.94(19)$ & $\mathrm{C} 161-\mathrm{C} 14-\mathrm{C} 13$ & $118.2(3)$ \\
\hline
\end{tabular}




\begin{tabular}{|c|c|c|c|}
\hline $\mathrm{C} 2-\mathrm{C} 3-\mathrm{H} 3 \mathrm{~A}$ & 109.2 & $\mathrm{C} 166-\mathrm{C} 161-\mathrm{C} 162$ & $117.5(3)$ \\
\hline $\mathrm{C} 4-\mathrm{C} 3-\mathrm{H} 3 \mathrm{~A}$ & 109.2 & $\mathrm{C} 166-\mathrm{C} 161-\mathrm{C} 14$ & $121.8(3)$ \\
\hline $\mathrm{C} 2-\mathrm{C} 3-\mathrm{H} 3 \mathrm{~B}$ & 109.2 & $\mathrm{C} 162-\mathrm{C} 161-\mathrm{C} 14$ & $120.3(3)$ \\
\hline $\mathrm{C} 4-\mathrm{C} 3-\mathrm{H} 3 \mathrm{~B}$ & 109.2 & $\mathrm{C} 163-\mathrm{C} 162-\mathrm{C} 161$ & $120.0(4)$ \\
\hline $\mathrm{H} 3 \mathrm{~A}-\mathrm{C} 3-\mathrm{H} 3 \mathrm{~B}$ & 107.9 & $\mathrm{C} 163-\mathrm{C} 162-\mathrm{H} 162$ & 120.0 \\
\hline $\mathrm{O} 4-\mathrm{C} 4-\mathrm{C} 5$ & $105.72(18)$ & $\mathrm{C} 161-\mathrm{C} 162-\mathrm{H} 162$ & 120.0 \\
\hline $\mathrm{O} 4-\mathrm{C} 4-\mathrm{C} 3$ & $113.48(19)$ & $\mathrm{C} 162-\mathrm{C} 163-\mathrm{C} 164$ & $122.1(4)$ \\
\hline $\mathrm{C} 5-\mathrm{C} 4-\mathrm{C} 3$ & $108.75(19)$ & $\mathrm{C} 162-\mathrm{C} 163-\mathrm{H} 163$ & 119.0 \\
\hline $\mathrm{O} 4-\mathrm{C} 4-\mathrm{H} 4$ & 109.6 & $\mathrm{C} 164-\mathrm{C} 163-\mathrm{H} 163$ & 119.0 \\
\hline $\mathrm{C} 5-\mathrm{C} 4-\mathrm{H} 4$ & 109.6 & $\mathrm{C} 165-\mathrm{C} 164-\mathrm{C} 163$ & $115.2(3)$ \\
\hline $\mathrm{C} 3-\mathrm{C} 4-\mathrm{H} 4$ & 109.6 & $\mathrm{C} 165-\mathrm{C} 164-\mathrm{C} 181$ & $124.3(3)$ \\
\hline $\mathrm{C} 6-\mathrm{C} 5-\mathrm{C} 4$ & $111.3(2)$ & $\mathrm{C} 163-\mathrm{C} 164-\mathrm{C} 181$ & $120.2(3)$ \\
\hline $\mathrm{C} 6-\mathrm{C} 5-\mathrm{H} 5 \mathrm{~A}$ & 109.4 & $\mathrm{C} 164-\mathrm{C} 165-\mathrm{C} 166$ & $123.3(5)$ \\
\hline $\mathrm{C} 4-\mathrm{C} 5-\mathrm{H} 5 \mathrm{~A}$ & 109.4 & $\mathrm{C} 164-\mathrm{C} 165-\mathrm{H} 165$ & 118.3 \\
\hline $\mathrm{C} 6-\mathrm{C} 5-\mathrm{H} 5 \mathrm{~B}$ & 109.4 & $\mathrm{C} 166-\mathrm{C} 165-\mathrm{H} 165$ & 118.3 \\
\hline $\mathrm{C} 4-\mathrm{C} 5-\mathrm{H} 5 \mathrm{~B}$ & 109.4 & $\mathrm{C} 161-\mathrm{C} 166-\mathrm{C} 165$ & $121.3(5)$ \\
\hline $\mathrm{H} 5 \mathrm{~A}-\mathrm{C} 5-\mathrm{H} 5 \mathrm{~B}$ & 108.0 & $\mathrm{C} 161-\mathrm{C} 166-\mathrm{H} 166$ & 119.3 \\
\hline $\mathrm{N} 1-\mathrm{C} 6-\mathrm{C} 5$ & $111.49(19)$ & $\mathrm{C} 165-\mathrm{C} 166-\mathrm{H} 166$ & 119.3 \\
\hline $\mathrm{N} 1-\mathrm{C} 6-\mathrm{H} 6 \mathrm{~A}$ & 109.3 & $\mathrm{C} 173-\mathrm{C} 172-\mathrm{H} 172$ & 119.0 \\
\hline $\mathrm{C} 5-\mathrm{C} 6-\mathrm{H} 6 \mathrm{~A}$ & 109.3 & $\mathrm{C} 172-\mathrm{C} 173-\mathrm{H} 173$ & 120.1 \\
\hline $\mathrm{N} 1-\mathrm{C} 6-\mathrm{H} 6 \mathrm{~B}$ & 109.3 & $\mathrm{C} 176-\mathrm{C} 175-\mathrm{H} 175$ & 118.8 \\
\hline $\mathrm{C} 5-\mathrm{C} 6-\mathrm{H} 6 \mathrm{~B}$ & 109.3 & $\mathrm{C} 175-\mathrm{C} 176-\mathrm{H} 176$ & 118.7 \\
\hline $\mathrm{H} 6 \mathrm{~A}-\mathrm{C} 6-\mathrm{H} 6 \mathrm{~B}$ & 108.0 & $\mathrm{C} 183-\mathrm{C} 181-\mathrm{C} 182$ & $109.0(3)$ \\
\hline $\mathrm{C} 41-\mathrm{O} 4-\mathrm{C} 4$ & $116.44(17)$ & $\mathrm{C} 183-\mathrm{C} 181-\mathrm{C} 164$ & $112.3(2)$ \\
\hline $\mathrm{O} 4-\mathrm{C} 41-\mathrm{C} 151$ & $112.52(19)$ & $\mathrm{C} 182-\mathrm{C} 181-\mathrm{C} 164$ & $109.4(2)$ \\
\hline $\mathrm{O} 4-\mathrm{C} 41-\mathrm{C} 141$ & $106.93(18)$ & $\mathrm{C} 183-\mathrm{C} 181-\mathrm{C} 184$ & $107.5(2)$ \\
\hline $\mathrm{C} 151-\mathrm{C} 41-\mathrm{C} 141$ & $112.66(18)$ & $\mathrm{C} 182-\mathrm{C} 181-\mathrm{C} 184$ & $108.4(2)$ \\
\hline $\mathrm{O} 4-\mathrm{C} 41-\mathrm{H} 41$ & 108.2 & $\mathrm{C} 164-\mathrm{C} 181-\mathrm{C} 184$ & $110.1(2)$ \\
\hline $\mathrm{C} 151-\mathrm{C} 41-\mathrm{H} 41$ & 108.2 & $\mathrm{C} 181-\mathrm{C} 182-\mathrm{H} 18 \mathrm{~A}$ & 109.5 \\
\hline $\mathrm{C} 141-\mathrm{C} 41-\mathrm{H} 41$ & 108.2 & $\mathrm{C} 181-\mathrm{C} 182-\mathrm{H} 18 \mathrm{~B}$ & 109.5 \\
\hline $\mathrm{C} 142-\mathrm{C} 141-\mathrm{C} 146$ & $117.8(2)$ & $\mathrm{H} 18 \mathrm{~A}-\mathrm{C} 182-\mathrm{H} 18 \mathrm{~B}$ & 109.5 \\
\hline $\mathrm{C} 142-\mathrm{C} 141-\mathrm{C} 41$ & $122.5(2)$ & $\mathrm{C} 181-\mathrm{C} 182-\mathrm{H} 18 \mathrm{C}$ & 109.5 \\
\hline $\mathrm{C} 146-\mathrm{C} 141-\mathrm{C} 41$ & $119.7(2)$ & $\mathrm{H} 18 \mathrm{~A}-\mathrm{C} 182-\mathrm{H} 18 \mathrm{C}$ & 109.5 \\
\hline $\mathrm{C} 141-\mathrm{C} 142-\mathrm{C} 143$ & $121.1(2)$ & $\mathrm{H} 18 \mathrm{~B}-\mathrm{C} 182-\mathrm{H} 18 \mathrm{C}$ & 109.5 \\
\hline $\mathrm{C} 141-\mathrm{C} 142-\mathrm{H} 142$ & 119.4 & $\mathrm{C} 181-\mathrm{C} 183-\mathrm{H} 18 \mathrm{D}$ & 109.5 \\
\hline $\mathrm{C} 143-\mathrm{C} 142-\mathrm{H} 142$ & 119.4 & $\mathrm{C} 181-\mathrm{C} 183-\mathrm{H} 18 \mathrm{E}$ & 109.5 \\
\hline $\mathrm{C} 144-\mathrm{C} 143-\mathrm{C} 142$ & $120.1(3)$ & $\mathrm{H} 18 \mathrm{D}-\mathrm{C} 183-\mathrm{H} 18 \mathrm{E}$ & 109.5 \\
\hline $\mathrm{C} 144-\mathrm{C} 143-\mathrm{H} 143$ & 120.0 & $\mathrm{C} 181-\mathrm{C} 183-\mathrm{H} 18 \mathrm{~F}$ & 109.5 \\
\hline $\mathrm{C} 142-\mathrm{C} 143-\mathrm{H} 143$ & 120.0 & $\mathrm{H} 18 \mathrm{D}-\mathrm{C} 183-\mathrm{H} 18 \mathrm{~F}$ & 109.5 \\
\hline $\mathrm{C} 145-\mathrm{C} 144-\mathrm{C} 143$ & $119.7(3)$ & $\mathrm{H} 18 \mathrm{E}-\mathrm{C} 183-\mathrm{H} 18 \mathrm{~F}$ & 109.5 \\
\hline $\mathrm{C} 145-\mathrm{C} 144-\mathrm{H} 144$ & 120.2 & $\mathrm{C} 181-\mathrm{C} 184-\mathrm{H} 18 \mathrm{G}$ & 109.5 \\
\hline $\mathrm{C} 143-\mathrm{C} 144-\mathrm{H} 144$ & 120.2 & $\mathrm{C} 181-\mathrm{C} 184-\mathrm{H} 18 \mathrm{H}$ & 109.5 \\
\hline $\mathrm{C} 144-\mathrm{C} 145-\mathrm{C} 146$ & $120.3(3)$ & $\mathrm{H} 18 \mathrm{G}-\mathrm{C} 184-\mathrm{H} 18 \mathrm{H}$ & 109.5 \\
\hline $\mathrm{C} 144-\mathrm{C} 145-\mathrm{H} 145$ & 119.9 & $\mathrm{C} 181-\mathrm{C} 184-\mathrm{H} 18 \mathrm{I}$ & 109.5 \\
\hline $\mathrm{C} 146-\mathrm{C} 145-\mathrm{H} 145$ & 119.9 & $\mathrm{H} 18 \mathrm{G}-\mathrm{C} 184-\mathrm{H} 18 \mathrm{I}$ & 109.5 \\
\hline $\mathrm{C} 145-\mathrm{C} 146-\mathrm{C} 141$ & $121.0(3)$ & $\mathrm{H} 18 \mathrm{H}-\mathrm{C} 184-\mathrm{H} 18 \mathrm{I}$ & 109.5 \\
\hline $\mathrm{C} 145-\mathrm{C} 146-\mathrm{H} 146$ & 119.5 & $\mathrm{C} 26-\mathrm{C} 21-\mathrm{C} 22$ & $119.2(2)$ \\
\hline $\mathrm{C} 141-\mathrm{C} 146-\mathrm{H} 146$ & 119.5 & $\mathrm{C} 26-\mathrm{C} 21-\mathrm{C} 27$ & $120.1(2)$ \\
\hline
\end{tabular}




\begin{tabular}{|c|c|c|c|}
\hline $\mathrm{C} 152-\mathrm{C} 151-\mathrm{C} 156$ & $118.5(2)$ & $\mathrm{C} 22-\mathrm{C} 21-\mathrm{C} 27$ & $120.7(2)$ \\
\hline $\mathrm{C} 152-\mathrm{C} 151-\mathrm{C} 41$ & $120.3(2)$ & $\mathrm{C} 23-\mathrm{C} 22-\mathrm{C} 21$ & $119.6(2)$ \\
\hline $\mathrm{C} 156-\mathrm{C} 151-\mathrm{C} 41$ & $121.2(2)$ & $\mathrm{C} 23-\mathrm{C} 22-\mathrm{H} 22$ & 120.2 \\
\hline $\mathrm{C} 153-\mathrm{C} 152-\mathrm{C} 151$ & $121.4(3)$ & $\mathrm{C} 21-\mathrm{C} 22-\mathrm{H} 22$ & 120.2 \\
\hline $\mathrm{C} 153-\mathrm{C} 152-\mathrm{H} 152$ & 119.3 & $\mathrm{C} 24-\mathrm{C} 23-\mathrm{C} 22$ & $122.4(2)$ \\
\hline $\mathrm{C} 151-\mathrm{C} 152-\mathrm{H} 152$ & 119.3 & $\mathrm{C} 24-\mathrm{C} 23-\mathrm{N} 23$ & $118.5(2)$ \\
\hline $\mathrm{C} 154-\mathrm{C} 153-\mathrm{C} 152$ & $119.7(3)$ & $\mathrm{C} 22-\mathrm{C} 23-\mathrm{N} 23$ & $119.2(2)$ \\
\hline $\mathrm{C} 154-\mathrm{C} 153-\mathrm{H} 153$ & 120.2 & $\mathrm{C} 25-\mathrm{C} 24-\mathrm{C} 23$ & $117.0(2)$ \\
\hline $\mathrm{C} 152-\mathrm{C} 153-\mathrm{H} 153$ & 120.2 & $\mathrm{C} 25-\mathrm{C} 24-\mathrm{H} 24$ & 121.5 \\
\hline $\mathrm{C} 153-\mathrm{C} 154-\mathrm{C} 155$ & $120.0(3)$ & $\mathrm{C} 23-\mathrm{C} 24-\mathrm{H} 24$ & 121.5 \\
\hline $\mathrm{C} 153-\mathrm{C} 154-\mathrm{H} 154$ & 120.0 & $\mathrm{C} 24-\mathrm{C} 25-\mathrm{C} 26$ & $122.6(2)$ \\
\hline $\mathrm{C} 155-\mathrm{C} 154-\mathrm{H} 154$ & 120.0 & $\mathrm{C} 24-\mathrm{C} 25-\mathrm{N} 25$ & $117.6(2)$ \\
\hline $\mathrm{C} 156-\mathrm{C} 155-\mathrm{C} 154$ & $120.4(3)$ & $\mathrm{C} 26-\mathrm{C} 25-\mathrm{N} 25$ & $119.8(2)$ \\
\hline $\mathrm{C} 156-\mathrm{C} 155-\mathrm{H} 155$ & 119.8 & $\mathrm{C} 21-\mathrm{C} 26-\mathrm{C} 25$ & $119.3(2)$ \\
\hline $\mathrm{C} 154-\mathrm{C} 155-\mathrm{H} 155$ & 119.8 & $\mathrm{C} 21-\mathrm{C} 26-\mathrm{H} 26$ & 120.3 \\
\hline $\mathrm{C} 155-\mathrm{C} 156-\mathrm{C} 151$ & $120.1(3)$ & $\mathrm{C} 25-\mathrm{C} 26-\mathrm{H} 26$ & 120.3 \\
\hline $\mathrm{C} 155-\mathrm{C} 156-\mathrm{H} 156$ & 120.0 & $\mathrm{O} 21-\mathrm{C} 27-\mathrm{O} 22$ & $127.0(2)$ \\
\hline $\mathrm{C} 151-\mathrm{C} 156-\mathrm{H} 156$ & 120.0 & $\mathrm{O} 21-\mathrm{C} 27-\mathrm{C} 21$ & $118.1(2)$ \\
\hline $\mathrm{N} 1-\mathrm{C} 11-\mathrm{C} 12$ & $114.0(2)$ & $\mathrm{O} 22-\mathrm{C} 27-\mathrm{C} 21$ & $115.0(2)$ \\
\hline $\mathrm{N} 1-\mathrm{C} 11-\mathrm{H} 11 \mathrm{~A}$ & 108.8 & $\mathrm{O} 24-\mathrm{N} 23-\mathrm{O} 23$ & $124.5(3)$ \\
\hline $\mathrm{C} 12-\mathrm{C} 11-\mathrm{H} 11 \mathrm{~A}$ & 108.8 & $\mathrm{O} 24-\mathrm{N} 23-\mathrm{C} 23$ & $117.9(3)$ \\
\hline $\mathrm{N} 1-\mathrm{C} 11-\mathrm{H} 11 \mathrm{~B}$ & 108.8 & $\mathrm{O} 23-\mathrm{N} 23-\mathrm{C} 23$ & $117.7(2)$ \\
\hline $\mathrm{C} 12-\mathrm{C} 11-\mathrm{H} 11 \mathrm{~B}$ & 108.8 & $\mathrm{O} 26-\mathrm{N} 25-\mathrm{O} 25$ & $124.2(3)$ \\
\hline $\mathrm{H} 11 \mathrm{~A}-\mathrm{C} 11-\mathrm{H} 11 \mathrm{~B}$ & 107.7 & $\mathrm{O} 26-\mathrm{N} 25-\mathrm{C} 25$ & $117.6(3)$ \\
\hline $\mathrm{C} 13-\mathrm{C} 12-\mathrm{C} 11$ & $111.0(3)$ & $\mathrm{O} 25-\mathrm{N} 25-\mathrm{C} 25$ & $118.1(3)$ \\
\hline $\mathrm{C} 13-\mathrm{C} 12-\mathrm{H} 12 \mathrm{~A}$ & 104.2 & & \\
\hline $\mathrm{C} 11-\mathrm{N} 1-\mathrm{C} 2-\mathrm{C} 3$ & $-179.77(19)$ & $\mathrm{O} 14-\mathrm{C} 14-\mathrm{C} 161-\mathrm{C} 166$ & $10.3(6)$ \\
\hline $\mathrm{C} 6-\mathrm{N} 1-\mathrm{C} 2-\mathrm{C} 3$ & $-56.5(2)$ & $\mathrm{C} 13-\mathrm{C} 14-\mathrm{C} 161-\mathrm{C} 166$ & $-170.9(5)$ \\
\hline $\mathrm{N} 1-\mathrm{C} 2-\mathrm{C} 3-\mathrm{C} 4$ & $57.2(2)$ & $\mathrm{O} 14-\mathrm{C} 14-\mathrm{C} 161-\mathrm{C} 162$ & $-162.5(4)$ \\
\hline $\mathrm{C} 2-\mathrm{C} 3-\mathrm{C} 4-\mathrm{O} 4$ & $61.4(2)$ & $\mathrm{C} 13-\mathrm{C} 14-\mathrm{C} 161-\mathrm{C} 162$ & $16.4(5)$ \\
\hline $\mathrm{C} 2-\mathrm{C} 3-\mathrm{C} 4-\mathrm{C} 5$ & $-55.9(2)$ & $\mathrm{C} 166-\mathrm{C} 161-\mathrm{C} 162-\mathrm{C} 163$ & $5.6(7)$ \\
\hline $\mathrm{O} 4-\mathrm{C} 4-\mathrm{C} 5-\mathrm{C} 6$ & $-66.3(2)$ & $\mathrm{C} 14-\mathrm{C} 161-\mathrm{C} 162-\mathrm{C} 163$ & $178.6(4)$ \\
\hline $\mathrm{C} 3-\mathrm{C} 4-\mathrm{C} 5-\mathrm{C} 6$ & $55.8(3)$ & $\mathrm{C} 161-\mathrm{C} 162-\mathrm{C} 163-\mathrm{C} 164$ & $0.8(7)$ \\
\hline $\mathrm{C} 2-\mathrm{N} 1-\mathrm{C} 6-\mathrm{C} 5$ & $57.0(2)$ & $\mathrm{C} 162-\mathrm{C} 163-\mathrm{C} 164-\mathrm{C} 165$ & $-6.2(8)$ \\
\hline $\mathrm{C} 11-\mathrm{N} 1-\mathrm{C} 6-\mathrm{C} 5$ & $-178.8(2)$ & $\mathrm{C} 162-\mathrm{C} 163-\mathrm{C} 164-\mathrm{C} 181$ & $-179.9(4)$ \\
\hline $\mathrm{C} 4-\mathrm{C} 5-\mathrm{C} 6-\mathrm{N} 1$ & $-57.7(3)$ & $\mathrm{C} 163-\mathrm{C} 164-\mathrm{C} 165-\mathrm{C} 166$ & $5.7(11)$ \\
\hline $\mathrm{C} 5-\mathrm{C} 4-\mathrm{O} 4-\mathrm{C} 41$ & $-179.83(19)$ & $\mathrm{C} 181-\mathrm{C} 164-\mathrm{C} 165-\mathrm{C} 166$ & $179.0(6)$ \\
\hline $\mathrm{C} 3-\mathrm{C} 4-\mathrm{O} 4-\mathrm{C} 41$ & $61.1(3)$ & $\mathrm{C} 162-\mathrm{C} 161-\mathrm{C} 166-\mathrm{C} 165$ & $-6.3(10)$ \\
\hline $\mathrm{C} 4-\mathrm{O} 4-\mathrm{C} 41-\mathrm{C} 151$ & $53.7(3)$ & $\mathrm{C} 14-\mathrm{C} 161-\mathrm{C} 166-\mathrm{C} 165$ & $-179.2(6)$ \\
\hline $\mathrm{C} 4-\mathrm{O} 4-\mathrm{C} 41-\mathrm{C} 141$ & $177.88(18)$ & $\mathrm{C} 164-\mathrm{C} 165-\mathrm{C} 166-\mathrm{C} 161$ & $0.5(13)$ \\
\hline $\mathrm{O} 4-\mathrm{C} 41-\mathrm{C} 141-\mathrm{C} 142$ & $4.6(3)$ & $\mathrm{C} 165-\mathrm{C} 164-\mathrm{C} 181-\mathrm{C} 183$ & $-24.9(6)$ \\
\hline $\mathrm{C} 151-\mathrm{C} 41-\mathrm{C} 141-\mathrm{C} 142$ & $128.7(2)$ & $\mathrm{C} 163-\mathrm{C} 164-\mathrm{C} 181-\mathrm{C} 183$ & $148.2(4)$ \\
\hline $\mathrm{O} 4-\mathrm{C} 41-\mathrm{C} 141-\mathrm{C} 146$ & $-176.5(2)$ & $\mathrm{C} 165-\mathrm{C} 164-\mathrm{C} 181-\mathrm{C} 182$ & $96.3(6)$ \\
\hline $\mathrm{C} 151-\mathrm{C} 41-\mathrm{C} 141-\mathrm{C} 146$ & $-52.4(3)$ & $\mathrm{C} 163-\mathrm{C} 164-\mathrm{C} 181-\mathrm{C} 182$ & $-90.6(4)$ \\
\hline $\mathrm{C} 146-\mathrm{C} 141-\mathrm{C} 142-\mathrm{C} 143$ & $1.4(4)$ & $\mathrm{C} 165-\mathrm{C} 164-\mathrm{C} 181-\mathrm{C} 184$ & $-144.6(6)$ \\
\hline $\mathrm{C} 41-\mathrm{C} 141-\mathrm{C} 142-\mathrm{C} 143$ & $-179.7(2)$ & $\mathrm{C} 163-\mathrm{C} 164-\mathrm{C} 181-\mathrm{C} 184$ & $28.4(4)$ \\
\hline $\mathrm{C} 141-\mathrm{C} 142-\mathrm{C} 143-\mathrm{C} 144$ & $-0.2(4)$ & $\mathrm{C} 26-\mathrm{C} 21-\mathrm{C} 22-\mathrm{C} 23$ & $1.4(3)$ \\
\hline
\end{tabular}




$\begin{array}{llll}\mathrm{C} 142-\mathrm{C} 143-\mathrm{C} 144-\mathrm{C} 145 & -1.2(4) & \mathrm{C} 27-\mathrm{C} 21-\mathrm{C} 22-\mathrm{C} 23 & -176.3(2) \\ \mathrm{C} 143-\mathrm{C} 144-\mathrm{C} 145-\mathrm{C} 146 & 1.4(5) & \mathrm{C} 21-\mathrm{C} 22-\mathrm{C} 23-\mathrm{C} 24 & -0.9(4) \\ \mathrm{C} 144-\mathrm{C} 145-\mathrm{C} 146-\mathrm{C} 141 & -0.1(5) & \mathrm{C} 21-\mathrm{C} 22-\mathrm{C} 23-\mathrm{N} 23 & 178.1(2) \\ \mathrm{C} 142-\mathrm{C} 141-\mathrm{C} 146-\mathrm{C} 145 & -1.3(4) & \mathrm{C} 22-\mathrm{C} 23-\mathrm{C} 24-\mathrm{C} 25 & 0.0(4) \\ \mathrm{C} 41-\mathrm{C} 141-\mathrm{C} 146-\mathrm{C} 145 & 179.8(3) & \mathrm{N} 23-\mathrm{C} 23-\mathrm{C} 24-\mathrm{C} 25 & -179.0(2) \\ \mathrm{O} 4-\mathrm{C} 41-\mathrm{C} 151-\mathrm{C} 152 & -129.7(2) & \mathrm{C} 23-\mathrm{C} 24-\mathrm{C} 25-\mathrm{C} 26 & 0.4(4) \\ \mathrm{C} 141-\mathrm{C} 41-\mathrm{C} 151-\mathrm{C} 152 & 109.3(2) & \mathrm{C} 23-\mathrm{C} 24-\mathrm{C} 25-\mathrm{N} 25 & -179.6(2) \\ \mathrm{O} 4-\mathrm{C} 41-\mathrm{C} 151-\mathrm{C} 156 & 50.5(3) & \mathrm{C} 22-\mathrm{C} 21-\mathrm{C} 26-\mathrm{C} 25 & -1.0(3) \\ \mathrm{C} 141-\mathrm{C} 41-\mathrm{C} 151-\mathrm{C} 156 & -70.5(3) & \mathrm{C} 27-\mathrm{C} 21-\mathrm{C} 26-\mathrm{C} 25 & 176.7(2) \\ \mathrm{C} 156-\mathrm{C} 151-\mathrm{C} 152-\mathrm{C} 153 & -0.6(4) & \mathrm{C} 24-\mathrm{C} 25-\mathrm{C} 26-\mathrm{C} 21 & 0.1(4) \\ \mathrm{C} 41-\mathrm{C} 151-\mathrm{C} 152-\mathrm{C} 153 & 179.6(2) & \mathrm{N} 25-\mathrm{C} 25-\mathrm{C} 26-\mathrm{C} 21 & -180.0(2) \\ \mathrm{C} 151-\mathrm{C} 152-\mathrm{C} 153-\mathrm{C} 154 & -0.2(4) & \mathrm{C} 26-\mathrm{C} 21-\mathrm{C} 27-\mathrm{O} 21 & 1.3(3) \\ \mathrm{C} 152-\mathrm{C} 153-\mathrm{C} 154-\mathrm{C} 155 & 0.6(5) & \mathrm{C} 22-\mathrm{C} 21-\mathrm{C} 27-\mathrm{O} 21 & 179.0(2) \\ \mathrm{C} 153-\mathrm{C} 154-\mathrm{C} 155-\mathrm{C} 156 & -0.3(5) & \mathrm{C} 26-\mathrm{C} 21-\mathrm{C} 27-\mathrm{O} 22 & -177.2(2) \\ \mathrm{C} 154-\mathrm{C} 155-\mathrm{C} 156-\mathrm{C} 151 & -0.5(4) & \mathrm{C} 22-\mathrm{C} 21-\mathrm{C} 27-\mathrm{O} 22 & 0.5(3) \\ \mathrm{C} 152-\mathrm{C} 151-\mathrm{C} 156-\mathrm{C} 155 & 0.9(4) & \mathrm{C} 24-\mathrm{C} 23-\mathrm{N} 23-\mathrm{O} 24 & -4.2(4) \\ \mathrm{C} 41-\mathrm{C} 151-\mathrm{C} 156-\mathrm{C} 155 & -179.3(2) & \mathrm{C} 22-\mathrm{C} 23-\mathrm{N} 23-\mathrm{O} 24 & 176.8(2) \\ \mathrm{C} 2-\mathrm{N} 1-\mathrm{C} 11-\mathrm{C} 12 & \mathrm{C} 24-\mathrm{C} 23-\mathrm{N} 23-\mathrm{O} 23 & 174.9(2) \\ \mathrm{C} 6-\mathrm{N} 1-\mathrm{C} 11-\mathrm{C} 12 & \mathrm{C} 222-4(3) & \mathrm{C} 24-\mathrm{C} 25-\mathrm{N} 25-\mathrm{O} 26 & -4.1(4) \\ \mathrm{N} 1-\mathrm{C} 11-\mathrm{C} 12-\mathrm{C} 13 & \mathrm{C} 26-\mathrm{C} 25-\mathrm{N} 25-\mathrm{O} 26 & -11.3(4) \\ \mathrm{C} 11-\mathrm{C} 12-\mathrm{C} 13-\mathrm{C} 14 & -122.9(3) & \mathrm{C} 24-\mathrm{C} 25-\mathrm{N} 25-\mathrm{O} 25 & -9.9(4) \\ \mathrm{C} 12-\mathrm{C} 13-\mathrm{C} 14-\mathrm{O} 14 & -179.2(3) & \mathrm{C} 26-\mathrm{C} 25-\mathrm{N} 25-\mathrm{O} 25 & 170.2(2) \\ \mathrm{C} 12-\mathrm{C} 13-\mathrm{C} 14-\mathrm{C} 161 & 179.5(3) & & \\ \end{array}$

Hydrogen-bond geometry $\left(\AA,{ }^{\circ}\right)$

\begin{tabular}{lllll}
\hline$D-\mathrm{H} \cdots A$ & $D-\mathrm{H}$ & $\mathrm{H} \cdots A$ & $D \cdots A$ & $D-\mathrm{H} \cdots A$ \\
\hline $\mathrm{N} 1-\mathrm{H} 1 \cdots \mathrm{O} 22$ & $0.99(3)$ & $1.66(2)$ & $2.634(3)$ & $167(2)$ \\
$\mathrm{C} 2-\mathrm{H} 2 A \cdots \mathrm{O} 25^{\mathrm{i}}$ & 0.97 & 2.50 & $3.444(3)$ & 163 \\
$\mathrm{C} 11-\mathrm{H} 11 A \cdots \mathrm{O} 14^{\mathrm{ii}}$ & 0.97 & 2.49 & $3.358(4)$ & 150 \\
\hline
\end{tabular}

Symmetry codes: (i) $x-1,-y+3 / 2, z+1 / 2$; (ii) $x-1, y, z$. 